\title{
A Distributed Power Sharing Approach for Islanded Microgrids
}

\author{
Tuan V. Hoang and Hong-Hee Lee \\ School of Electrical Engineering \\ University of Ulsan, Korea \\ Phone number:+82-52-259-2187, e-mail: hoangtuan0404@gmail.com, hhlee@ mail.ulsan.ac.kr
}

\begin{abstract}
This paper presents a distributed control scheme to accurately share active, reactive, and selected harmonic power demands for islanded droop-controlled microgrids. The proposed strategy requires only a sparse communication network because the DG unit uses local and its neighbours' information to carry out the control algorithm. In the proposed strategy, virtual impedances at selected dominant frequencies are adaptively regulated based on the consensus algorithm, and the power sharing errors in the islanded microgrid system is eliminated irrespective of load conditions. The proposed method can be implemented directly in practice without any information such as the feeder impedances or the detailed microgrid configuration. The simulation results are also provided to validate the feasibility and effectiveness of the proposed method.
\end{abstract}

\section{Key words}

Droop control, distributed generation (DG), microgrid, distributed power sharing.

\section{Introduction}

With the growth of environmental awareness and carbon emissions concerns, distributed generations (DGs) such as photovoltaic, wind turbines, fuel cells, microturbines, etc., have been widely installed in the power distribution systems in recent years. To realize effectively coordinated operation of multiple parallel DG units, the microgrid concept has been widely adopted because the microgrid can operate flexibly either in a gridconnected or in an islanded mode to provide a costeffective operation and more reliable power to the loads and customers [1].

In the islanded operation, it is important to share the load power demands by multiple DG units according to their power ratings. Conventionally, the real powerfrequency $(P-\omega)$ and reactive power-voltage magnitude $(Q-E)$ droop controllers are applied to facilitate the power sharing requirement in a decentralized manner [2]-[4]. However, it has been pointed out that the droop controller cannot realize the accurate reactive and harmonic power sharing due to the mismatch in feeder impedances even though the accurate active power sharing is always guaranteed at the steady state [5]-[7].

To cope with the inaccurate reactive and harmonic power sharing problems, many improved decentralized

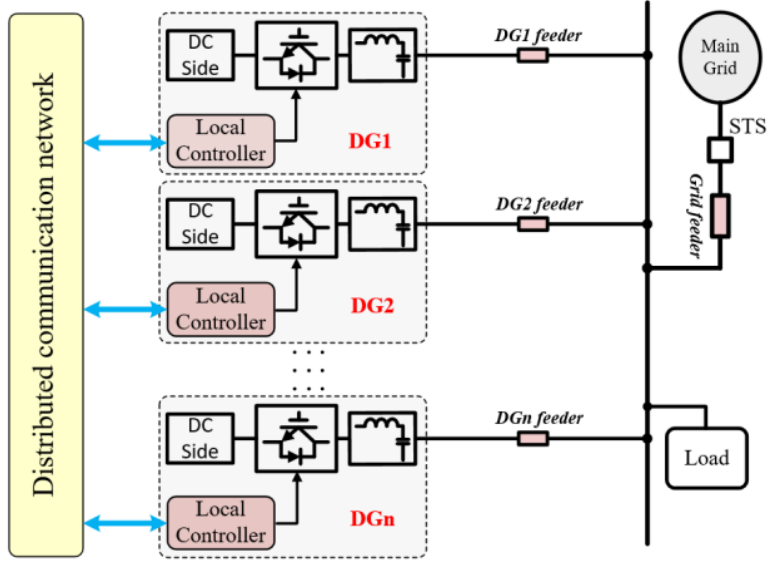

Fig. 1. Configuration of the islanded microgrid.

methods have been reported. Among them, the virtual impedance-based approach has been one of the most attractive solutions due to its capability of solving the inaccurate power sharing problem and the power control instability at the same time [8], [9]. However, this method usually requires the information about DG feeder impedances. As we know, the load conditions and the microgrid structure change frequently and randomly due to the "plug-and-play" character of loads and DG units, and it is very hard to detect the line impedances in realtime which are key information for the virtual impedance-based methods. Therefore, it is difficult to achieve the accurate reactive and harmonic power sharing in decentralized way, especially when the load condition or microgrid configuration changes. To overcome this problem, many communication-aided methods have been proposed to enhance the power sharing performance based on the centralized approach and distributed approach.

Even though the centralized-based methods (CBMs) have early adopted to realize accurate reactive power sharing [10], [11] and harmonic power sharing [12], [13] without using the line impedance information, the CBMs are sensitive to a failure of MGCC or single-point communication link. To remove this problem, the distributed-based methods (DBMs) have been proposed 


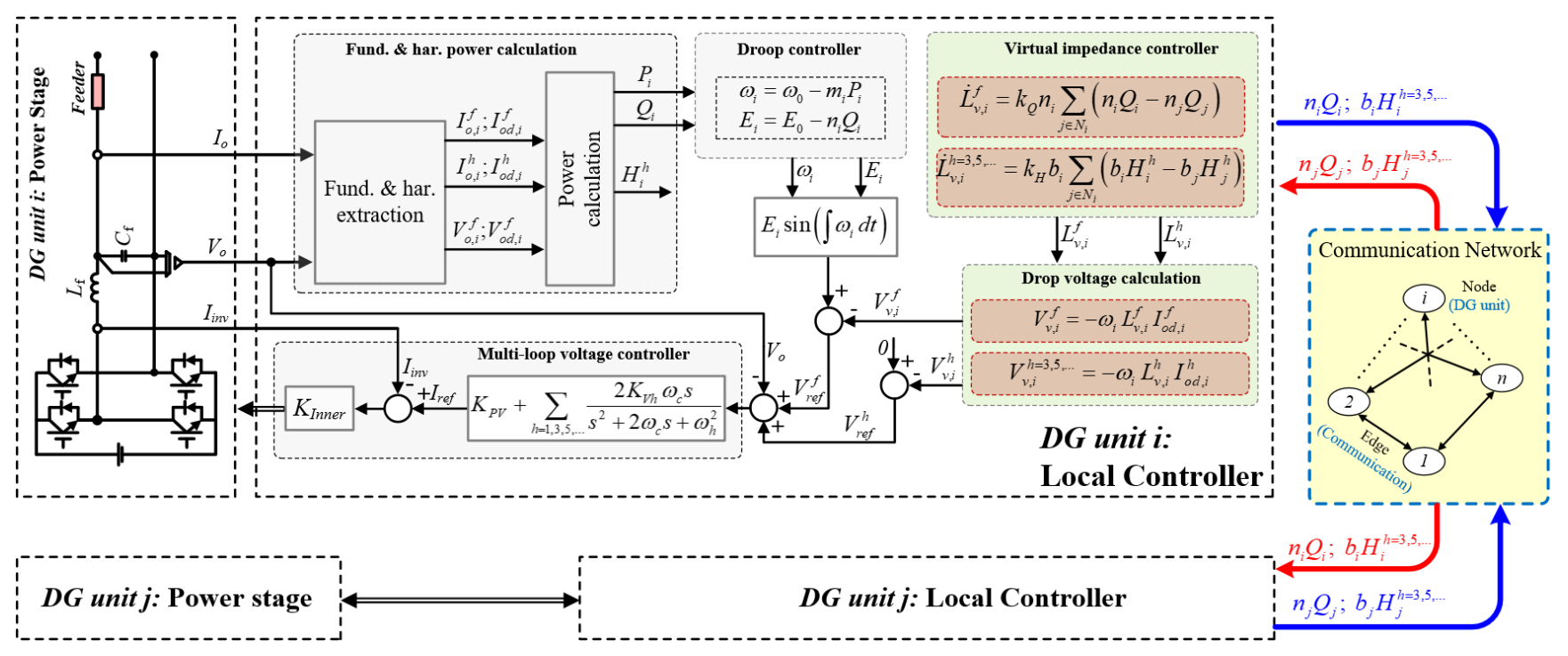

Fig. 2. Proposed power control scheme for the $i$-th DG unit.

recently [14]. By using only a sparse communication network, many distributed power sharing methods have been reported recently [15]-[17]. However, because most of them are realized based on DG output voltage magnitude regulation, the distributed virtual impedance control is not fulfilled sufficiently. Even though accurate reactive and harmonic power sharing are realized by means of a consensus-based virtual impedance control approach in [18], the virtual impedance regulator becomes very complicated due to many control gains.

In this paper, we propose a distributed power sharing strategy for islanded microgrids to provide accurate active, reactive, and selected harmonic power sharing. A distributed virtual impedance control based on the consensus algorithm is developed to deal with the inaccurate power sharing problem in islanded microgrids. The proposed methodology can be directly realized without any information about feeder impedances, load powers, and microgrid structure. The simulation results are provided to verify the effectiveness of the proposed method.

\section{Islanded Microgrid Operation}

Fig. 1 illustrates a typical islanded microgrid configuration composed of several power electronicsbased DG units and loads. Each DG unit includes a DC source, inverter, LC filter, and a local controller. Also, to optimize the operation of microgrid system, each DG unit communicates with its neighbours through distributed communication network. As shown in Fig. 1, the microgrid can operate in grid-connected mode or islanded mode by controlling the static transfer switch (STS).

In islanded operation, DG units are conventionally controlled by $P-\omega$ and $Q-E$ droop controllers:

$$
\begin{aligned}
& \omega_{i}=\omega_{0}-m_{i} P_{i}, \\
& E_{i}=E_{0}-n_{i} Q_{i},
\end{aligned}
$$

where $\omega_{0}$ and $E_{0}$ are the nominal values of DG angular frequency and DG voltage magnitude, respectively; $P_{\mathrm{i}}$ and $Q_{\mathrm{i}}$ are measured real and reactive powers, respectively; $m_{\mathrm{i}}$ and $n_{\mathrm{i}}$ are real and reactive power droop coefficients of $i$ th DG unit, respectively.

With the derived angular frequency and voltage magnitude from (1) and (2), the instantaneous voltage reference $V_{\text {droop,i }}$ is given in (3) [10]:

$$
V_{\text {droop }, i}=E_{i} \sin \left(\int \omega_{i} d t\right) \text {. }
$$

\section{Proposed Control Scheme}

\section{A. Communication Network Model}

To carry out a distributed control strategy, a distributed communication network, whose graphic symbol is shown in the right-hand side of Fig. 2, is usually adopted. Based on the graph theory [19], in this study, the communication network is modelled as an undirected and connected graph $\Gamma=(\xi, \lambda)$ with a nonempty finite set of $n$ DG nodes $\xi=\{1,2, \cdots, n\}$, and a set of undirected edges $\lambda \subseteq \xi \times \xi$. If there is a communication link between two nodes $i$ and $j$, where $(i, j) \in \lambda$, these two nodes are called as communication neighbours. All the neighbours of $i$-th DG unit constitute its neighbour set $N_{\mathrm{i}}$ $=\{j \in \xi \mid(i, j) \in \lambda\}$, and $\left|N_{\mathrm{i}}\right|$ is denoted as its cardinality. The adjacency matrix of graph $\Gamma$ is matrix $\Lambda$, where $\Lambda=$ $\left(a_{i j}\right)_{\mathrm{n} \times \mathrm{n}}$ with $a_{i j}=1$ if $(i, j) \in \lambda$, otherwise $a_{i j}=0$. For ease analysis, we assume that there is no self-edge in the graph, i.e., $a_{i i}=0 \forall i \in \xi$. The degree matrix is denoted by $D=\operatorname{diag}\left(d_{i}\right)_{n \times n}$ with $d_{i}=\sum_{j \in N i} a_{i j}$. Then, by using these definitions, the Laplacian matrix $\mathscr{L}=\left(l_{i j}\right)_{\mathrm{n} \times \mathrm{n}}$ of graph $\Gamma$ is given by $\mathscr{L}=D-\Lambda$.

It should be emphasized that $\mathscr{L}$ is a symmetric and positive semidefinite matrix. Furthermore, for an undirected and connected graph, zero is simple eigenvalue of its Laplacian matrix $\mathscr{L}$. And, the corresponding right eigenvector to this simple zero eigenvalue is $\underline{1}_{\mathrm{n}}=\left[\begin{array}{llll}1 & 1 & \cdots & 1\end{array}\right]^{\mathrm{T}}$, i.e., $\mathscr{L} \underline{1}_{\mathrm{n}}=0$.

\section{B. Proposed Control Scheme}

Using the distributed communication network, the complete diagram of the proposed strategy for islanded microgrids is shown in Fig. 2, where the control scheme 




Fig. 3. Decomposition of fundamental and harmonic components ( $\mathrm{T}$ denotes the fundamental cycle time).

TABLE I

ELECTRICAL AND CONTROL PARAMETERS

\begin{tabular}{|c|c|c|c|}
\hline \multicolumn{4}{|c|}{ Electrical Setup } \\
\hline System parameter & Symbol & \multicolumn{2}{|l|}{ Value } \\
\hline Filter Capacitance & $C_{\mathrm{f}}$ & \multicolumn{2}{|l|}{$10 \mu \mathrm{F}$} \\
\hline Filter Inductance & $L_{\mathrm{f}}$ & \multicolumn{2}{|l|}{$1.3 \mathrm{mH}$} \\
\hline DC Link Voltage & $V_{\mathrm{dc}}$ & \multicolumn{2}{|l|}{$280 \mathrm{~V}$} \\
\hline Nominal Operating Voltage & $E_{0}$ & \multicolumn{2}{|c|}{$110 \mathrm{~V}, 60 \mathrm{~Hz}$} \\
\hline Switching Frequency & $f_{\mathrm{sw}}$ & \multicolumn{2}{|c|}{$10 \mathrm{kHz}$} \\
\hline \multicolumn{4}{|c|}{ Control Parameters } \\
\hline Parameter & Symbol & DGs $1 \& 2$ & DG 3 \\
\hline$P-\omega$ Doop Coeff. & $m_{\mathrm{i}}(\mathrm{rad} / \mathrm{W} \cdot \mathrm{s})$ & 0.0015 & 0.003 \\
\hline$Q-V$ Doop Coeff. & $n_{\mathrm{i}}(\mathrm{V} / \mathrm{Var})$ & 0.02 & 0.04 \\
\hline M-PR Coeff. of Outer Loop & $K_{P V} / K_{V h}$ & $0.1 / 40$ & $0.1 / 40$ \\
\hline Cutoff Freq. of Outer Loop & $\omega_{c}(\mathrm{Rad} / \mathrm{s})$ & 12.5 & 12.5 \\
\hline P Coeff. of Inner Loop & $K_{I N N E R}$ & 25 & 25 \\
\hline
\end{tabular}

includes three main parts: power calculation, consensusbased virtual impedance control to regulate the power sharing, and multiloop voltage controller to output desired DG voltage.

\section{1) DG Unit Power Calculation:}

In order to calculate the active, reactive, and harmonic powers of $i$-th DG unit, the fundamental and harmonic components of $i$-th DG line current and output voltage should be extracted, whose simplified decomposition principle is sketched in Fig. 3. As shown in Fig. 3, the fundamental line current and output voltage component ( $I_{o}^{f}$ and $V_{o}^{f}$ ) and harmonic line current components $\left(I_{o}^{h}\right)$ are detected through the second-order generalized integrator (SOGI) [20], and their corresponding conjugated signal $I_{o d}^{f}, V_{o d}^{f}$, and $I_{o d}^{h}$ are obtained by delaying $I_{o}^{f}$, $V_{o}^{f}$, and $I_{o}^{h}$ for a quarter fundamental and $h$-order harmonic cycle, respectively [12]. Then, the active and reactive powers of $i$-th DG unit are calculated as following:

$$
\begin{aligned}
& P_{i}=\frac{\omega_{L P F}}{2\left(s+\omega_{L P F}\right)}\left(V_{o, i}^{f} I_{o, i}^{f}+V_{o d, i}^{f} I_{o d, i}^{f}\right), \\
& Q_{i}=\frac{\omega_{L P F}}{2\left(s+\omega_{L P F}\right)}\left(V_{o d, i}^{f} I_{o, i}^{f}-V_{o, i}^{f} I_{o d, i}^{f}\right),
\end{aligned}
$$

where $\omega_{L P F}$ is the cutoff frequency of the low-pass filter.

And, the $h$-order harmonic power of $i$-th DG unit is calculated based on the approximation of $V_{o, r m s}^{f}=E_{0} / \sqrt{2}$ and the $h$-order harmonic line current and its conjugated signal ( $I_{o, i}^{h}$ and $I_{o d, i}^{h}$, respectively) [12]

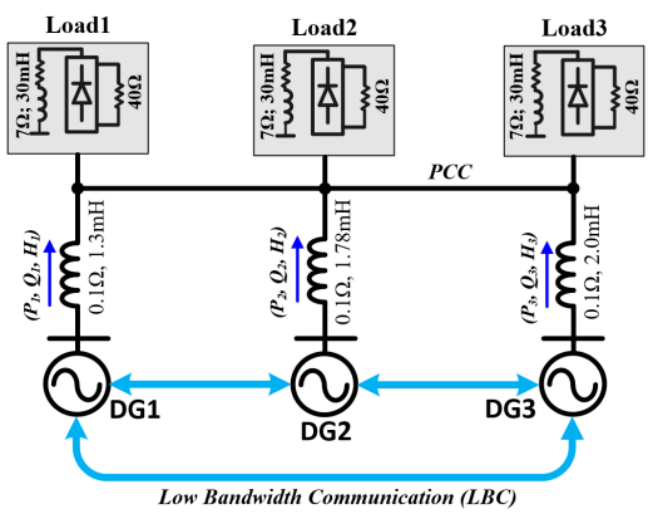

Fig. 4. Islanded microgrid used in simulation.

$$
H_{i}^{h=3,5, \ldots}=V_{o r m s, i}^{f} I_{o r m s, i}^{h}=\frac{1}{2} E_{0} \sqrt{\left(I_{o, i}^{h}\right)^{2}+\left(I_{o d, i}^{h}\right)^{2}},
$$

where $V_{\text {orms }, i}^{f}$ and $I_{\text {orms }, i}^{h}$ are the rms values of the $i$-th DG unit fundamental voltage and $h$-order harmonic line current, respectively.

\section{2) Power Sharing Control:}

In order to achieve accurate reactive power sharing $\left(n_{i} Q_{i}=n_{j} Q_{j}\right)$ by means of the consensus control, the $n_{i} Q_{i}$ should be chosen as DG state [19]. However, in the consensus control, selected DG state is required to be directly controllable, but $n_{i} Q_{i}$ cannot do it. To cope with this problem, the controllable quantity $\dot{L}_{v, i}^{f}$ is used instead of $n_{i} \dot{Q}_{i}$ to regulate the $i$-th DG fundamental virtual impedance based on the following equation:

$$
\dot{L}_{v, i}^{f}=k_{Q} n_{i} \sum_{j \in N_{i}}\left(n_{i} Q_{i}-n_{j} Q_{j}\right),
$$

where $k_{\mathrm{Q}}$ is a positive control gain.

From (7), the update rule of $i$-th DG virtual impedance is expressed as following:

$$
L_{v, i}^{f}=k_{Q} n_{i} \int \sum_{j \in N_{i}}\left(n_{i} Q_{i}-n_{j} Q_{j}\right) .
$$

And, the drop voltage on the DG virtual impedance $V_{v, i}^{f}$ is calculated in (9):

$$
V_{v, i}^{f}=-\left(\omega_{i} L_{v, i}^{f}\right) I_{o d, i}^{f} .
$$

Similarly, the $h$-order harmonic virtual impedance of the $i$-th DG unit $L_{v, i}^{h}$ is adaptively regulated to eliminate the respective harmonic power sharing errors based on the relationship in (10), and its voltage drop is calculated from (11):

$$
\begin{gathered}
\dot{L}_{v, i}^{h}=k_{H} b_{i} \sum_{j \in N_{i}}\left(b_{i} Q_{i}-b_{j} Q_{j}\right), \\
V_{v, i}^{h}=-\left(h \omega_{i} L_{v, i}^{h}\right) I_{o d, i}^{h},
\end{gathered}
$$

where $b_{\mathrm{i}}$ is the harmonic power sharing factor defined by

$$
b_{i}^{h}=\frac{1}{H_{\text {rated }, i}^{h}},
$$

where $H_{\text {rated }, i}^{h}(i=1,2, \cdots, n)$ is the $h$-order harmonic power rating of $i$-th DG unit.

Then, the reference voltage for the multiloop voltage controller is modified as following: 



Fig. 5. Simulated power sharing performance: (a) Active power; (b) Reactive power; (c) Third harmonic power; (d) Fifth harmonic power.

$$
\begin{aligned}
V_{\text {ref }, i} & =V_{\text {doop }, i}-V_{v, i}^{f}-V_{v, i}^{h} \\
& =\underbrace{V_{\text {doop }, i}+\left(\omega_{i} L_{v, i}^{f}\right) I_{o d, i}}_{V_{r e f, i}^{f}}+\underbrace{\left(h \omega_{i} L_{v, i}^{h}\right) I_{o d, i}^{h}}_{V_{r e f, i}^{h}} .
\end{aligned}
$$

\section{3) Multiloop Voltage Controller:}

In order to control the inverter to generate the desired output voltage for the DG unit according to the voltage reference in (13), the multiloop voltage controller in Fig. 2 is adopted. In the multiloop voltage controller, the outer loop uses a proportional controller and multiple quasiresonant (M-PR) controllers with the following transfer function in [12]:

$$
G_{\text {Outer }}(s)=K_{P V}+\sum_{h=1,3,5, \ldots} \frac{2 K_{V h} \omega_{c} s}{s^{2}+2 \omega_{c} s+\omega_{h}^{2}},
$$

where $K_{\mathrm{PV}}$ and $K_{\mathrm{Vh}}$ are outer loop proportional and resonant controller gains, respectively, and $\omega_{\mathrm{c}}$ is the cutoff frequency of the resonant controllers. The inner loop is a simple proportional controller with control gain $K_{\text {Inner }}$ with inductor current feedback, which aims to provide sufficient damping to the output LC filter [10]:

$$
G_{\text {Inner }}(s)=K_{\text {Inner }} \text {. }
$$


Fig. 6. Variation of virtual impedances: (a) at fundamental frequency $(\Omega)$; (b) at third harmonic frequency $(\Omega)$; (c) at harmonic frequency $(\Omega)$.

\section{Simulation Validation}

As shown in Fig. 4, the islanded microgrid with three DG units is used to validate the proposed power sharing strategy with three loads 1-3 at PCC, and the rated powers of units 1 and 2 are twice that of unit 3 . Simulation is carried out with the aids of the PSIM software. The system parameters are listed in Table I, and a $1 \mathrm{~ms}$ delay is included in the communication links of DG units.

The power sharing performance with the proposed control method is shown in Fig. 5. Initially, the microgrid is operated with the conventional droop method with loads $1-3$, and the proposed method is applied at $t=5 \mathrm{~s}$. In order to verify the effectiveness of the proposed method in case of step load changes, Load2 is sequentially disconnected from and connected to the system at $10 \mathrm{~s}$ and $15 \mathrm{~s}$, respectively.

As shown in Fig. 5, even though the active power demands are always shared properly among DG units, the reactive and harmonic powers are not accurately shared with conventional droop method in the stage 1 . However, once the proposed method is applied from $5 \mathrm{~s}$, the total reactive and harmonic load powers are shared accurately by DG units after small transient interval. The accurate reactive power sharing and harmonic power sharing are remained continuously irrespective of the load power demand change in stage 3. Furthermore, the proposed control scheme always shows perfect active power sharing in the steady-state.

Fig. 6 shows the variations of the DG virtual impedances for each stage in Fig. 5: after the proposed 
method is applied at $t=5 \mathrm{~s}$, the fundamental, third, and fifth harmonic virtual impedances are regulated adaptively [see Figs. 6(a)-(c), respectively]. Consequently, the perfect active, reactive, and harmonic power sharing are achieved regardless of load condition as clearly verified in Fig. 5.

\section{Conclusion}

In this paper, an adaptive virtual impedance control approach has been proposed by means of the consensus algorithm to achieve accurate power sharing for islanded microgrids. With the proposed method, the active, reactive, and selected harmonic power sharing are always guaranteed irrespective of load conditions. The virtual impedance control can be implemented directly in practice without any information about physical feeder impedance, detailed microgrid configuration, and load powers. Furthermore, the proposed approach uses only a sparse communication network which is less sensitive to the communication link failure. Therefore, the system becomes more reliable. Simulation results are also provided to validate the feasibility and effectiveness of the proposed control method.

\section{Acknowledgement}

This work was supported by the National Research Foundation of Korea Grant funded by the Korea Government under Grant NRF-2018R1D1A1 A09081779.

\section{References}

[1] A. Mehrizi-Sani and R. Iravani, "Potential-function based control of a microgrid in islanded and grid-connected modes," IEEE Trans. Power Syst., vol. 25, no. 4, pp. 18831891, 2010.

[2] J. M. Guerrero, J. Matas, L. G. D. V. De Vicuna, M. Castilla, and J. Miret, "Wireless-Control Strategy for Parallel Operation of Distributed-Generation Inverters," IEEE Trans. Ind. Electron., vol. 53, no. 5, pp. 1461-1470, Oct. 2006.

[3] K. De Brabandere, B. Bolsens, J. Van den Keybus, A. Woyte, J. Driesen, and R. Belmans, "A Voltage and Frequency Droop Control Method for Parallel Inverters," IEEE Trans. Power Electron., vol. 22, no. 4, pp. 1107-1115, Jul. 2007.

[4] J. C. Vasquez, R. A. Mastromauro, J. M. Guerrero, and M. Liserre, "Voltage support provided by a droop-controlled multifunctional inverter," IEEE Trans. Ind. Electron., vol. 56, no. 11, pp. 4510-4519, 2009.

[5] J. He and Y. W. Li, "Analysis, design, and implementation of virtual impedance for power electronics interfaced distributed generation," IEEE Trans. Ind. Appl., vol. 47, no. 6, pp. 2525-2538, 2011.

[6] H. Han, Y. Liu, Y. Sun, M. Su, and J. M. Guerrero, "An Improved Droop Control Strategy for Reactive Power Sharing in Islanded Microgrid," IEEE Trans. Power Electron., vol. 30, no. 6, pp. 3133-3141, Jun. 2015.

[7] J. He, Y. W. Li, and F. Blaabjerg, "An Enhanced Islanding Microgrid Reactive Power, Imbalance Power, and Harmonic Power Sharing Scheme," IEEE Trans. Power Electron., vol. 30, no. 6, pp. 3389-3401, Jun. 2015.

[8] P. Sreekumar and V. Khadkikar, "A new virtual harmonic impedance scheme for harmonic power sharing in an islanded microgrid," in 2016 IEEE Power and Energy Society General Meeting (PESGM), 2016, p. 1.
[9] P. Sreekumar and V. Khadkikar, "Direct Control of the Inverter Impedance to Achieve Controllable Harmonic Sharing in the Islanded Microgrid," IEEE Trans. Ind. Electron., vol. 64, no. 1, pp. 827-837, Jan. 2017.

[10] T. V. Hoang and H. H. Lee, "An Adaptive Virtual Impedance Control Scheme to Eliminate the ReactivePower-Sharing Errors in an Islanding Meshed Microgrid," IEEE J. Emerg. Sel. Top. Power Electron., vol. 6, no. 2, pp. 966-976, 2018.

[11] H. Mahmood, D. Michaelson, and J. Jiang, "Accurate Reactive Power Sharing in an Islanded Microgrid Using Adaptive Virtual Impedances," IEEE Trans. Power Electron., vol. 30, no. 3, pp. 1605-1617, Mar. 2015.

[12] T. V Hoang and H. Lee, "Accurate Power Sharing with Harmonic Power for Islanded Multibus Microgrids," IEEE J. Emerg. Sel. Top. Power Electron., p. 1, 2018.

[13] H. Moussa et al., "Harmonic Power Sharing With Voltage Distortion Compensation of Droop Controlled Islanded Microgrids," IEEE Trans. Smart Grid, vol. 9, no. 5, pp. 5335-5347, 2018.

[14] Y. Han, H. Li, P. Shen, E. A. A. Coelho, and J. M. Guerrero, "Review of Active and Reactive Power Sharing Strategies in Hierarchical Controlled Microgrids," IEEE Trans. Power Electron., vol. 32, no. 3, pp. 2427-2451, Mar. 2017.

[15] J. W. Simpson-Porco, Q. Shafiee, F. Dörfler, J. C. Vasquez, J. M. Guerrero, and F. Bullo, "Secondary Frequency and Voltage Control of Islanded Microgrids via Distributed Averaging," IEEE Trans. Ind. Electron., vol. 62, no. 11, pp. 7025-7038, Nov. 2015.

[16] J. Schiffer, T. Seel, J. Raisch, and T. Sezi, "Voltage Stability and Reactive Power Sharing in Inverter-Based Microgrids With Consensus-Based Distributed Voltage Control," IEEE Trans. Control Syst. Technol., vol. 24, no. 1, pp. 96-109, Jan. 2016.

[17] Q. Shafiee et al., "A Multi-Functional Fully Distributed Control Framework for AC Microgrids," IEEE Trans. Smart Grid, vol. 9, no. 4, pp. 3247-3258, 2018.

[18] J. Zhou, S. Kim, H. Zhang, and Q. Sun, "Consensus-Based Distributed Control for Accurate Reactive , Harmonic , and Imbalance Power Sharing in Microgrids," IEEE Trans. Smart Grid, vol. 9, no. 4, pp. 2453-2467, 2018.

[19] R. Olfati-Saber and R. M. Murray, "Consensus problems in networks of agents with switching topology and timedelays," IEEE Trans. Automat. Contr., vol. 49, no. 9, pp. 1520-1533, Sep. 2004.

[20] P. Rodriguez, A. Luna, I. Candela, R. Mujal, R. Teodorescu, and F. Blaabjerg, "Multiresonant FrequencyLocked Loop for Grid Synchronization of Power Converters Under Distorted Grid Conditions," IEEE Trans. Ind. Electron., vol. 58, no. 1, pp. 127-138, Jan. 2011. 